\title{
In-Body Propagation at ISM and UWB Frequencies for Abdominal Monitoring Applications
}

\author{
Mariella Särestöniemi \\ Centre for Wireless \\ Communications \\ Department of Information \\ Technology and Electrical \\ Engineering \\ University of Oulu, Finland
}

\author{
Carlos Pomalaza-Raez \\ Department of Electrical and \\ Computer Engineering \\ Purdue University \\ USA
}

\author{
Kamran Sayrafian \\ Information Technology \\ Laboratory \\ National Institute of Standards \\ and Technology \\ USA
}

\author{
Jari Iinatti \\ Centre for Wireless \\ Communications \\ Department of Information \\ Technology \\ and Electrical Engineering \\ University of Oulu, Finland
}

\begin{abstract}
This paper presents the results of an in-body propagation study for abdominal implant communication using a bio-matched mini-horn antenna. The wireless signal coverage in the abdomen area at various ISM and UWB frequency bands are measured using electromagnetic simulations with a 3D anatomical voxel model. Power flow analysis is conducted to visualize propagation within the abdominal tissues at several frequencies. As an example, S-Parameters of the channel between a capsule endoscopy pill and an on-body receiver has been studied and channel attenuations at different frequencies are compared. Using the directional bio-matched antenna, it is observed that at $915 \mathrm{MHz}$ the signal coverage in a horizontal plane is sufficient to almost include the whole small intestine. However, the depth of the coverage reduces substantially at higher frequency bands. This comparative analysis can provide some insight on the applicability of these frequencies for various abdominal monitoring applications. It can also provide some information about the required number and placement of the on-body receivers in order to ensure adequate signal reception.
\end{abstract}

Keywords- Electromagnetic Simulation, Gastrointestinal Tract monitoring, Implant Communication, In-body RF propagation

\section{INTRODUCTION}

Design of wireless medical monitoring systems that involve implants require deep understanding of radio frequency signal propagation within the human body tissues. This has motivated researchers to study in-body propagation for a variety of medical applications in recent years [1-7]. Additionally, there have been much effort to develop appropriate implant and on-body antennas to enhance the communication link between such medical devices [8-12].

Selection of the appropriate frequency band is one of the key issues in wireless implant communications. This selection could depend on the nature of the application and parameters such as the required data rate and quality of service, device size and location, etc. There are several studies on wireless propagation from implants at different frequency bands: Industrial, Scientific and Medical (ISM), Wireless Medical Telemetry Service (WMTS), Ultra WideBand (UWB), and Medical Device Radiocommunications Service (MedRadio) [3-7]. There are trade-offs for using each band for medical monitoring applications involving implants or ingestible electronics. For instance, the propagation loss in the human body tissues increases significantly at higher frequencies; however, design of a very small and efficient antennas for miniature-sized implants is quite challenging at lower frequencies. In addition, lower frequencies (or equivalently higher wavelengths) could create difficulties for some applications requiring higher resolution, e.g., accurate in-body localization, detection of small tumors or brain hemorrhages, etc. For comparison, relative permittivity and wavelengths in different body tissues at several frequencies are presented in Table I [13]. The wide range of dielectric properties show how different body tissues can impact the speed and attenuation of radio frequency signals inside the human body.

The 2.4 to $2.5 \mathrm{GHz}$ within the ISM band has been considered for implant communications since it enables high data rates with manageable propagation loss for some applications. However, as there are many other personal devices operating at this unlicensed frequency band, the resulting interference may pose an issue for medical applications which require high reliability. Although higher frequency bands enable higher data rates, the significant propagation loss at such frequencies inside the body could be a challenge. A possible solution to mitigate this loss is the use of directional on-body antennas along with their careful onbody placement to maximize in-body signal penetration; and therefore, achieving better wireless link quality [1], [7]

In [7], the authors have studied in-body signal power distribution in the abdomen at $4 \mathrm{GHz}$ using two different directional antennas designed for $3.75-4.25 \mathrm{GHz}$ band. This paper extends this study to cover several other frequencies: $915 \mathrm{MHz}, 2.45 \mathrm{GHz}, 3.1 \mathrm{GHz}$ and $5.8 \mathrm{GHz}$. The study is carried out using a directional bio-matched mini-horn antenna [11] which is applicable for the selected frequencies. A modified version of this antenna has also been used for detecting brain hemorrhage in [15].

The main objective of this paper is to study the wireless signal coverage from a directional bio-matched on-body antenna inside the abdominal and intestine area at several different frequencies. Two-Dimensional power flow analysis is conducted to visualize RF propagation within the abdominal tissues at selected frequencies. As an example, S-Parameters of the channel between a capsule endoscopy pill and an onbody receiver has also been studied and channel attenuations at different frequencies are compared.

This paper is organized as follows: Section II presents the considered scenario describing the simulation setup, on-body and in-body antennas as well as the computational human body model used in this study. Section III provides the results of the power flow analysis. The S-Parameters of the communication channel between the implant and the on-body mini-horn antennas are presented in Section IV. Finally, Section $\mathrm{V}$ provides further discussions along with some concluding remarks. 
TABLE I. RELATIVE PERMITTIVITY AND WAVELENGHT AT VARIOUS HUMAN BODY TISSUES AND FREQUENCIES

\begin{tabular}{|c|c|c|c|c|}
\hline & \multicolumn{4}{|c|}{$\begin{array}{l}\text { Relative permitivity / wavelength }[\mathrm{m}] \text { at the selected } \\
\text { frequencies }\end{array}$} \\
\hline Tissue & $915 \mathrm{MHz}$ & $2.45 \mathrm{GHz}$ & $3.1 \mathrm{GHz}$ & $5.8 \mathrm{GHz}$ \\
\hline Skin & $41.3 / 0.05$ & $38.0 / 0.022$ & $37.36 / 0.015$ & $35.1 / 0.0086$ \\
\hline Fat & $5.46 / 0.14$ & $5.28 / 0.053$ & $5.21 / 0.04$ & $4.95 / 0.023$ \\
\hline Muscle & $55.0 / 0.04$ & $52.7 / 0.017$ & $51.94 / 0.013$ & $48.5 / 0.0075$ \\
\hline Small intestine & $59.4 / 0.04$ & $54.43 / 0.016$ & $53.19 / 0.013$ & $48.6 / 0.0072$ \\
\hline Colon & $57.8 / 0.04$ & $53.87 / 0.017$ & $52.7 / 0.013$ & $48.5 / 0.007$ \\
\hline
\end{tabular}

\section{Simulation SCENARIO}

This study is conducted using Simulia CST studio suite ${ }^{1}$ 3D electromagnetic simulation and analysis software which is based on Finite Integration Technique (FIT). A voxel-based computational human body model (referred to as Laura) corresponding to a lean female body has been used for simulations. In order to reduce computational complexity and speed up the execution time, only the abdomen area of the torso has been used for simulations as shown in Fig. 1a.

The mini-horn antenna is placed directly above the navel area at the center of the abdomen. There is no muscle tissue in this area; therefore, the antenna would be facing a less challenging propagation media as far as dielectric properties are concerned [7], [13]. The capsule is placed inside the small intestine at two different locations as shown in Figs. $1 \mathrm{~b}$ and 1c. The first location, named "location A", is the nearest possible location within the small intestine of the body model to the location of the mini-horn antenna on the abdomen. This location approximately creates the best propagation condition considering the channel between the capsule and the on-body device. The distance between the capsule and the on-body antennas in this case is $4 \mathrm{~cm}$. The second location, named "location B", is the furthest possible location within the small intestine considering a 2D horizontal plane crossing the abdomen. This location is intended to represent the most challenging propagation condition for this communication channel on the 2D plane. The direct distance between the capsule and the on-body antenna is $13 \mathrm{~cm}$ in this case.

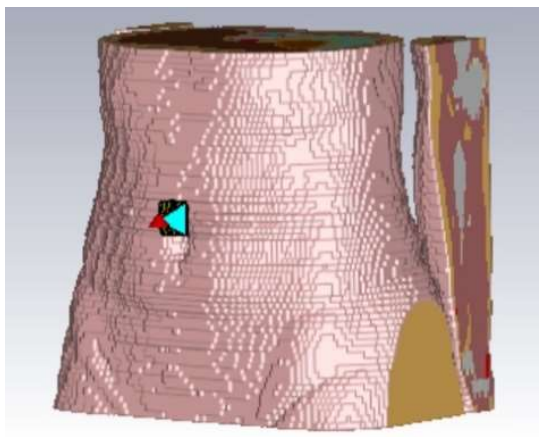

(a)

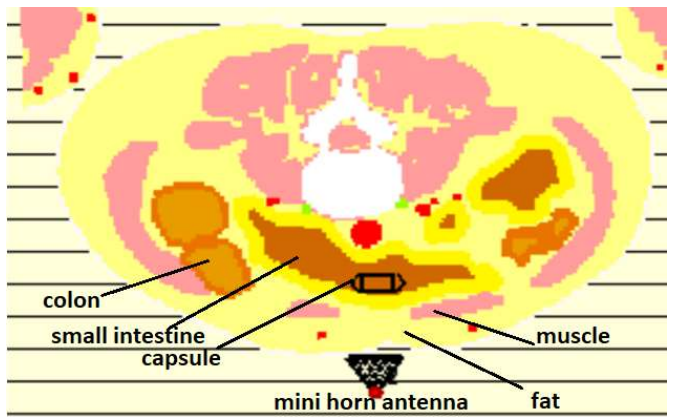

(b)

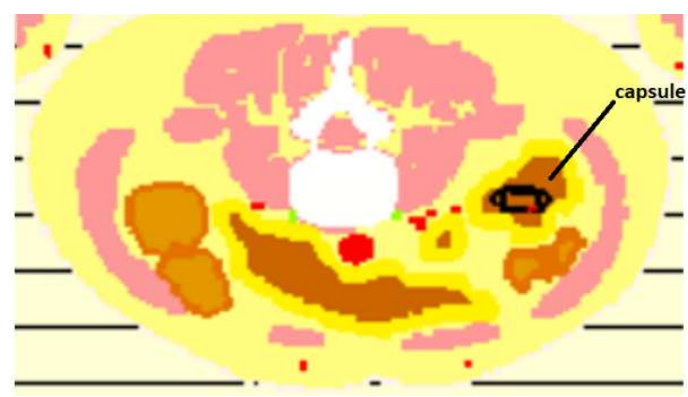

(c)

Fig. 1. a) Laura-voxel model, and cross-section of the voxel model with b) Capsule location A, and c) Capsule location B

\section{A. Bio-matched mini-horn antenna}

In this study, a directional bio-matched mini-horn antenna, shown in Fig. 2, is used for the evaluations. The antenna is based on the mini-horn antenna originally presented in [11]. It has been modified and remodeled for this study to obtain better matching with the voxel-based body model [15]. The modified structure has dimensions $h=2.7 \mathrm{~cm}$ and $d=1.8 \mathrm{~cm}$. The antenna is composed of water-filled holes which mimics the frequency-dependent relative permittivity of the underlying tissue over its entire bandwidth. The bio-matched mini-horn antenna has been designed to work while tightly attached to the surface of the skin. Therefore, some adjustments had to be done to ensure perfect contact with the surface of the voxel-based body model. Although the resolution of the Laura body model is high, pixelization on the abdomen surface could lead to air gaps between the antenna and the skin surface as shown in Fig. 2b. This will negatively impact the antenna matching and therefore the resulting measurements. To alleviate this problem, the air gaps have been replaced by very thin layers of skin and fat between the antenna and the surface of the body model where the antenna is placed. This is shown in Fig. 2c. These additional smoothing layers create a better and more realistic contact between the antenna and the abdomen surface.

The $\mathrm{S}_{11}$ parameter of the mini-horn antenna after the adjustment with smoothing layers is presented in Fig. 3. Further details of the antenna structure can be found in [11].

\footnotetext{
${ }^{1}$ Commercial products mentioned in this paper are merely intended to foster understanding. Their identification does not imply recommendation or endorsement by the National Institute of the Standards and Technology.
} 
a)

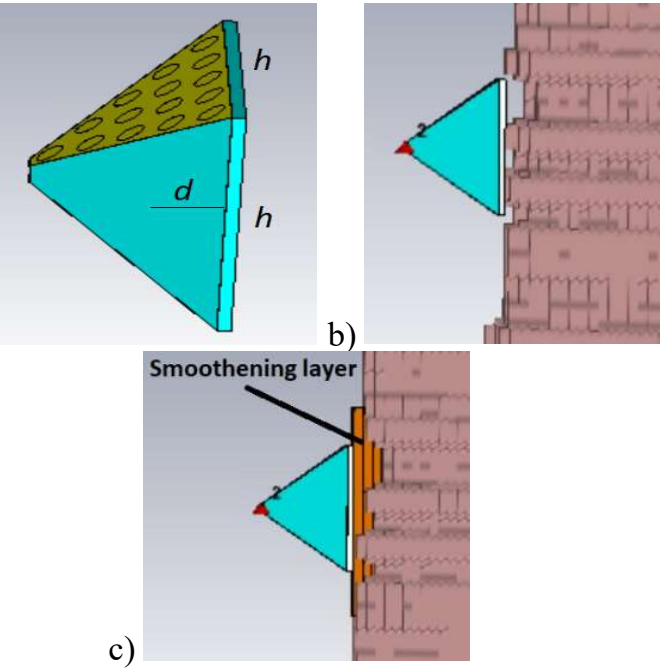

Fig. 2. a) A mini-horn on-body antenna designed for in-body communication, b) mini-horn antenna without smoothening layer, c) mini-horn with smoothening layer

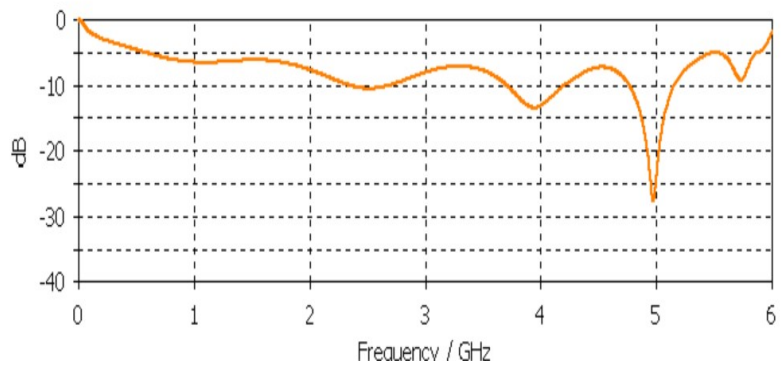

Fig. 3. S11 parameter of the mini-horn antenna while placed on the abdomen of the voxel model

\section{B. Capsule model}

The antenna used in the capsule model is a double-loop UWB antenna originally introduced in [10]. Although the antenna has been designed for 1-6 GHz frequency range, it can reasonably operate at the ISM (902-928) $\mathrm{MHz}$ band as well. The antenna is shown in Fig. 4a. The capsule model with the antenna embedded inside is presented in Fig. 4b. The dimensions of the capsule shell are comparable with the typical commercial capsules, i.e., $11 \mathrm{~mm} \times 25 \mathrm{~mm}$. The $\mathrm{S}_{11}$ parameter of the capsule antenna while inside the small intestine is presented in Fig. 5. Further details of the antenna structure, radiation patterns, as well as Specific Absorption Ratio (SAR) have been studied in [11].

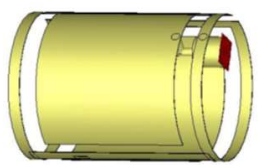

(a)

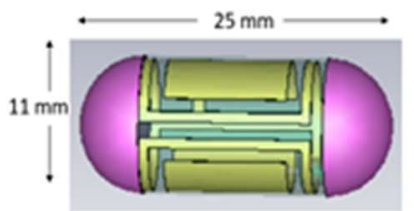

(b)
Fig. 4. a) UWB capsule antenna, b) Antenna inside the capsule shell

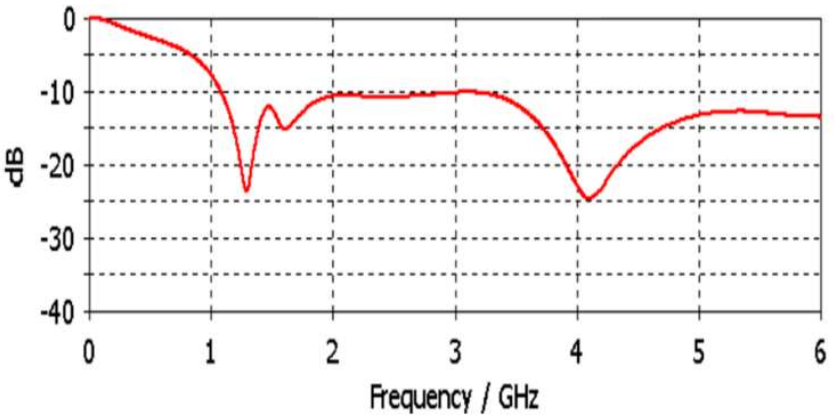

Fig. 5. S11 parameter of the capsule antenna inside the small intestine of the voxel model (capsule location A)

\section{Power Flow EVALuATIONS}

In this section, in-body propagation from the directional mini-horn antenna at different frequencies is studied using the notion of power flow [7]. Power flow is the time-averaged magnitude of the Poynting vector [14]. The Poynting vector represents the directional energy flux (the energy transfer per unit area per unit time) of an electromagnetic field. The flux of the Poynting Vector through a certain surface represents the total electromagnetic power flowing through it. Here, the power flow values (expressed as decibels) have been normalized so that $0 \mathrm{~dB}$ is the maximum, i.e., the value at the transmitting antenna.

Figs. 6a-d presents the power flow variation from the minihorn antenna (ranging from 0 to $-65 \mathrm{~dB}$ ) on a 2D horizontal plane crossing the abdomen area at $915 \mathrm{MHz}, 2.45 \mathrm{GHz}, 3.1$ $\mathrm{GHz}$, as well as $5.8 \mathrm{GHz}$, respectively. Sample power flow values at locations A and B are also provided in Table II. As will be seen in the next section, the $\mathrm{S}_{21}$ parameter at location $\mathrm{B}$ at $915 \mathrm{MHz}$ is $-62 \mathrm{~dB}$. This is a manageable channel attenuation to establish a wireless communication link between the capsule and the on-body antennas. The power flow value at location $B$ is $-65 \mathrm{~dB}$. Therefore, using this value as the threshold for the power flow plots in Figs. 6a-d can give us an approximate idea of RF coverage or signal penetration inside the abdomen area at various frequencies. For example, by calculating the average distance of the points on the contour representing $-65 \mathrm{~dB}$ in Figs. 6a-d to the location of the on-body antenna, one can find the estimate of the average coverage depth inside the abdomen within the $2 \mathrm{D}$ plane. In general, the 3D coverage of the directional mini horn antenna should be used to estimate the distance in 3D space. As observed in Fig. 6a, the power flow plot at $915 \mathrm{MHz}$ indicates good coverage of almost the whole small intestine on this horizontal cross section. The farthest part of the intestine incurs manageable loss for the design of a reliable communication link. At $2.45 \mathrm{MHz}$, as seen in Fig. $6 \mathrm{~b}$ and as expected, the power flow loss increases. At location B (power flow value of $-77 \mathrm{~dB}$ ), it will be harder to establish the communication link with the same reliability; therefore, a more sensitive receiver with a lower required signal-to-noise ratio would be needed. It should be noted that the antenna radiation pattern also changes at these frequencies. Changes in the beamwidth and/or the nulls in the antenna pattern will directly impact the power flow distribution and therefore signal coverage inside the abdomen.

At $3.1 \mathrm{GHz}$ and $5.8 \mathrm{GHz}$, the power flow plots show excessive loss inside the deepest parts of the intestine area even with the directional bio-matched antenna. At these frequencies, due to the limited signal propagation depth, more 
judicious placement or number of the on-body antennas are required to ensure the received signal is above the sensitivity threshold of the target receiver. Power flow plots for the vertical cross section of the abdomen have also been obtained for the frequencies under consideration but those results have been omitted for brevity. For capsule endoscopy application where coverage of the entire small intestine is needed, several on-body antennas should be used. Their optimal placements and achievability of $100 \%$ coverage for the small intestine is a problem that requires further investigation. For medical implants that are stationary, finding the location of an on-body receiver that is closer in distance is easier; however, clinical considerations might pose further constraints on proper onbody antenna placement.

a)
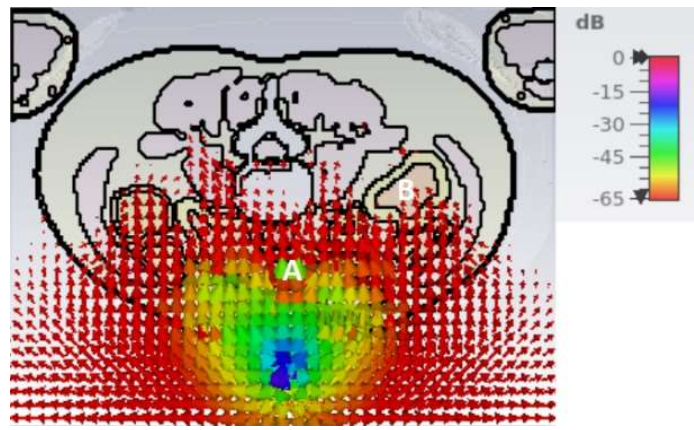

b)

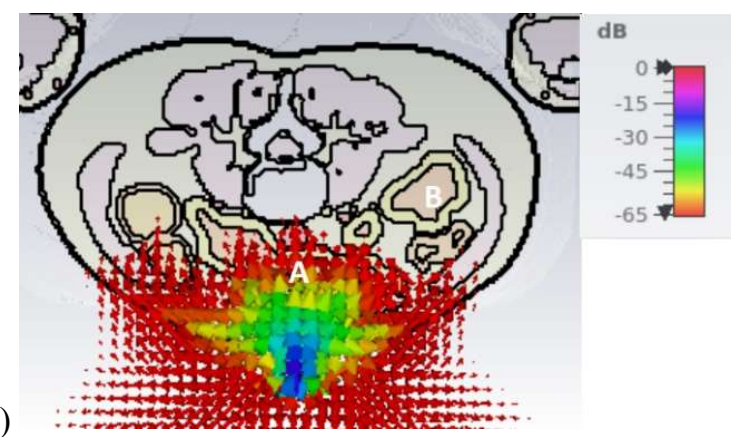

c)
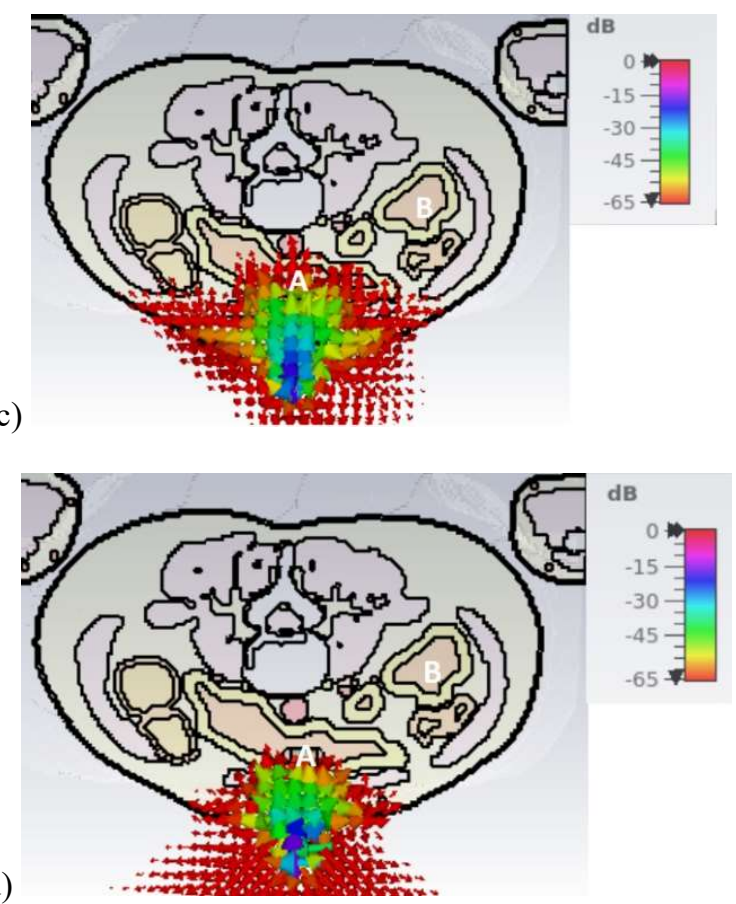

Fig. 6. Power flows at a) $915 \mathrm{MHz}$, b) $2.45 \mathrm{GHz}$, c) $3.1 \mathrm{GHz}$, d) $5.8 \mathrm{GHz}$
TABLE II: POWER FLOW VALUES IN DIFFERENT LOCATIONS AT DIFFERENT FREQUENCIES

\begin{tabular}{|c|c|c|}
\hline \multirow{2}{*}{$\begin{array}{c}\text { Frequency } \\
\text { [GHz] }\end{array}$} & \multicolumn{2}{|c|}{ Power flow value (dB) } \\
\cline { 2 - 3 } & Location $\boldsymbol{A}$ & Location $\boldsymbol{B}$ \\
\hline 0.915 & -46 & -65 \\
\hline 2.4 & -48 & -92 \\
\hline 3.1 & -49 & -100 \\
\hline 5.8 & -64 & -127 \\
\hline
\end{tabular}

\section{ChanNEL EVALUATION BETWEEN THE CAPSUlE} ENDOSCOPE AND THE ON-BODY RECEIVER

In this section, S-Parameters of the wireless channel between the mini-horn and the capsule antenna are evaluated for the two locations A and B. Figs. $7 \mathrm{a}$ and $7 \mathrm{~b}$ show parameters $S_{21}, S_{11}$ and $S_{22}$ for these two locations.

As observed, for location A, using the directional on-body antenna, the channel attenuation is moderate (i.e., around 40 $\mathrm{dB}$ ) within the entire range of the frequencies considered. This is the case even though the matching of the mini-horn antenna is not ideal at $915 \mathrm{MHz}$ (i.e., $\mathrm{S}_{11}=-6 \mathrm{~dB}$ ). At frequencies above $2 \mathrm{GHz}$, the antenna matching for the directional on-body antenna is much better (i.e., $\mathrm{S}_{11} \leq-10 \mathrm{~dB}$ ), and the forward transmission coefficient $S_{21}$ remains at an acceptable level across the range. In general, signal attenuation in the tissues increases as the frequency increases; however, variations in the capsule antenna matching (i.e., $\mathrm{S}_{22}$ ) and antenna radiation pattern, will impact the overall channel attenuation.

The S-Parameters for the wireless channel corresponding to location B is shown in Fig. 7b. Significant increase in channel attenuation versus frequency is more apparent for this location. At $915 \mathrm{MHz}$, the directional on-body antenna still results in manageable attenuation in order to have a reliable communication link. This does not seem to be the case for all other frequencies. The $\mathrm{S}_{21}$ values at different frequencies are summarized in Table III. The limited communication distance for on-body directional antennas necessitates additional receiver antennas at various locations of the abdomen to ensure proper capsule coverage and reliable communication for different segments of the intestine.

This study is conducted using only one on-body antenna to give an idea on the signal penetration depth at different frequencies. From the presented results, it can be concluded that a smaller number of on-body antennas are needed to provide full coverage of the abdominal area if lower frequencies are used. However, using higher frequency bands including $5.8 \mathrm{GHz}$ is also possible for gastrointestinal track monitoring if several on-body antennas are strategically placed around the abdomen (e.g., on the sides).

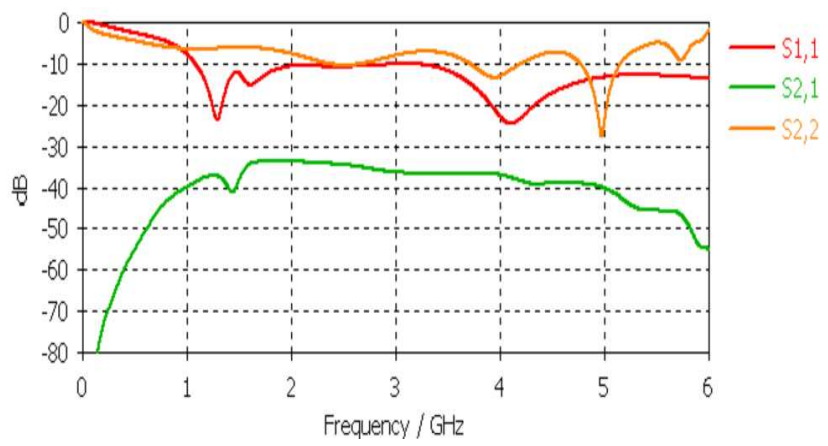


(a)

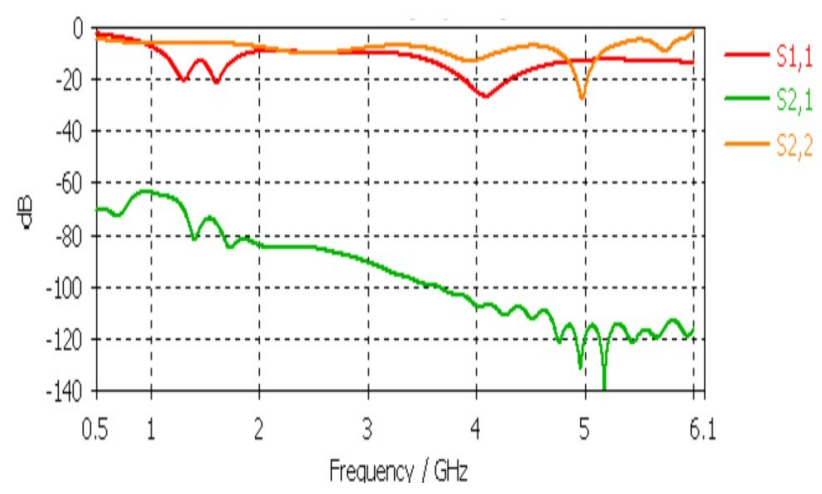

(b)

Fig. 7. S-parameters at a) capsule location A, b) capsule location B

TABLE III. $\mathrm{S}_{21}$ VALUES AT LOCATIONS A AND B

\begin{tabular}{|l|c|c|c|c|}
\hline & \multicolumn{4}{|c|}{$\mathbf{S}_{\mathbf{2 1}}$ values at the selected frequencies } \\
\hline $\begin{array}{l}\text { Frequency } \\
\text { Copsule }\end{array}$ & $915 \mathrm{MHz}$ & $2.45 \mathrm{GHz}$ & $3.1 \mathrm{GHz}$ & $5.8 \mathrm{GHz}$ \\
\hline Location & $-41 \mathrm{~dB}$ & $-35 \mathrm{~dB}$ & $-36 \mathrm{~dB}$ & $-47 \mathrm{~dB}$ \\
\hline Location A & $-62 \mathrm{~dB}$ & $-82 \mathrm{~dB}$ & $-90 \mathrm{~dB}$ & $-117 \mathrm{~dB}$ \\
\hline
\end{tabular}

\section{DISCUSSION AND CONLUSIONS}

Directional on-body antennas along with careful consideration of their on-body placement could enable utilization of higher frequency bands for implant communications. Although, directionality of these antennas implies deeper tissue penetration, the possibility of smaller 3D coverage might necessitate more numbers of such antennas to cover a certain area inside the human body, for example, coverage of the small intestine for wireless capsule endoscopy applications. Using higher frequencies will allow higher data rates for such applications. This translates to higher resolution images or even videos. Therefore, there is a trade-off between the achievable data rates and sufficient coverage for the entire small intestine. Properly designed directional antennas could assist in achieving full coverage; however, more investigations are necessary to better understand their feasibility in terms of the required number and their on-body placements. High directionality of onbody antennas could further assist in localization of capsule endoscopy inside the small intestine. This could further highlight the tradeoff between the location resolution and the number of required antennas. As mentioned before, clinical constraints for antenna placements could further complicate this problem.

The authors plan to continue this study by investigating the minimum number of antennas that could provide full coverage of the small intestine using several voxel-based body models having different sizes and shapes. Evaluation of the bit-error-rate (BER) performance of the impulse radio IRUWB-based receiver for the channels considered in this paper is also in our future research plans.

\section{ACKNOWLEDGMENT}

The work at the University of Oulu is supported by Academy of Finland 6Genesis Flagship (grant 318927) and the European Union's Horizon 2020 programme under the Marie Sklodowska-Curie grant agreement No. 872752. Mikko Linnanmäki from ExcellAnt is acknowledged for UWB capsule antenna's redesign. Mikko Parkkila and Uzman Ali from Radientum is acknowledged for mini-horn antenna re-design.

\section{REFERENCES}

[1] A. Teshome, B. Kibret, D. T. H. Lai, "A Review of Implant Communication Technology in WBAN, Progresses and Challenges," IEEE Reviews in Biomedical Engineering, 2018.

[2] R. B. Green, M. Hays, M. Mangino and E. Topsakal, "An Anatomical Model for the Simulation and Development of Subcutaneous Implantable Wireless Devices," in IEEE Transactions on Antennas and Propagation, vol. 68, no. 10, pp. 7170-7178, Oct. 2020

[3] M. Särestöniemi, C. Pomalaza-Ráez, C. Kissi, M. Berg, M. Hämäläinen and J. Iinatti, "WBAN Channel Characteristics Between Capsule Endoscope and Receiving Directional UWB On-Body Antennas," in IEEE Access, vol. 8, pp. 55953-55968, 2020.

[4] C. Andreu, C. Garcia-Pardo, S. Castelló-Palacios, A. Vallés-Lluch and N. Cardona, "Frequency Dependence of UWB In-Body Radio Channel Characteristics," in IEEE Microwave and Wireless Components Letters, vol. 28, no. 4, pp. 359-361, April 201

[5] S. Perez-Simbor, C. Andreu, C. Garcia-Pardo, M. Frasson and N. Cardona, "UWB Path Loss Models for Ingestible Devices," in IEEE Transactions on Antennas and Propagation, vol. 67, no. 8, pp. 50255034, Aug. 2019.

[6] P. A. Floor, R. Chávez-Santiago, A. N. Kim, K. Kansanen, T. A. Ramstad and I. Balasingham, "Communication Aspects for a Measurement Based UWB in-Body to on-Body Channel," in IEEE Access, vol. 7, pp. 29425-29440, 2019.

[7] M. Särestöniemi, C. Pomalaza-Raez, M. Berg, C. Kissi, M. Hämäläinen and J. Iinatti, "In-Body Power Distribution for Abdominal Monitoring and Implant Communications Systems," 2019 16th International Symposium on Wireless Communication Systems (ISWCS), Oulu, Finland, 2019, pp. 457-462

[8] N. A. Malik, P. Sant, T. Ajmal and M. Ur-Rehman, "Implantable Antennas for Bio-medical Applications," in IEEE Journal of Electromagnetics, RF and Microwaves in Medicine and Biology, 2021.

[9] S. Ma, T. Björninen, L. Sydänheimo, M. H. Voutilainen and L. Ukkonen, "Double Split Rings as Extremely Small and Tuneable Antennas for Brain Implantable Wireless Medical Microsystems," in IEEE Transactions on Antennas and Propagation, vol. 69, no. 2, pp. 760-768, Feb. 2021

[10] J. Shang and Y. Yu, "An Ultrawideband Capsule Antenna for Biomedical Applications," in IEEE Antennas and Wireless Propagation Letters, vol. 18, no. 12, pp. 2548-2551, Dec. 2019

[11] J. Blauert and A. Kiourti, "Bio-Matched Horn: A Novel 1-9 GHz OnBody Antenna for Low-Loss Biomedical Telemetry with Implants," in IEEE Transactions on Antennas and Propagation, vol. 67, no. 8, pp. 5054-5062, Aug. 2019.

[12] C. Kissi, M. Särestöniemi, C. Pomalaza-Raez, M. Sonkki, and M. N. Srifi, "Low-UWB directional antenna for Wireless Capsule Endoscopy localization," BodyNets2018.

[13] https://www.itis.ethz.ch/virtual-population/tissueproperties/databaseM (2021)

[14] S. J. Orfanidis, "Electromagnetic Waves and Antennas," 2002, revised 2016, online: http://www.ece.rutgers.edu/ orfanidi/ewa/.

[15] M. Särestöniemi, C. Pomalaza-Raez, J. Hakala, S. Myllymäki, J. Kilpijärvi, J. Iinatti, M. Hämäläinen, T. Myllylä,'’Detection of brain hemorrhage in white matter using analysis of radio channel characteristics," BodyNets2020, Oct. 2020. 